\title{
Ján Andres
}

Boundedness of solutions of the third order differential equation with oscillatory restoring and forcing terms

Czechoslovak Mathematical Journal, Vol. 36 (1986), No. 1, 1-6

Persistent URL: http://dml.cz/dmlcz/102058

\section{Terms of use:}

(C) Institute of Mathematics AS CR, 1986

Institute of Mathematics of the Czech Academy of Sciences provides access to digitized documents strictly for personal use. Each copy of any part of this document must contain these Terms of use.

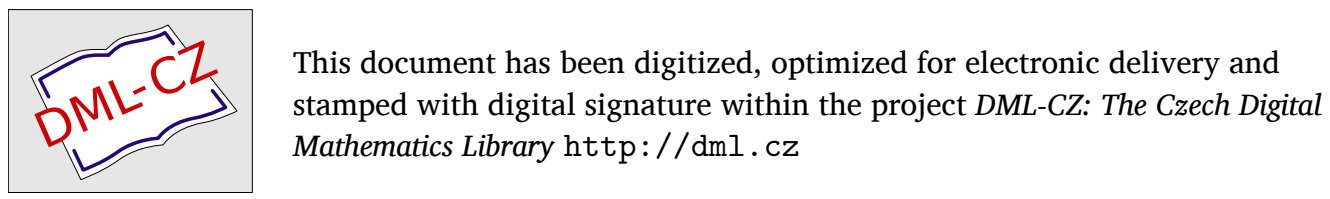




\section{BOUNDEDNESS OF SOLUTIONS OF THE THIRD ORDER DIFFERENTIAL EQUATION WITH OSCILLATORY RESTORING AND FORCING TERMS}

JAN ANDRES, Olomouc

(Received December 2, 1983)

1. In this paper we study the behaviour of solutions of the equation

$$
x^{\prime \prime \prime}+a x^{\prime \prime}+b x^{\prime}+h(x)=p^{\prime}(t),
$$

where $a>0, b>0$ are constants with $a^{2}>4 b$, the functions $h(x), p(t)$ have their first derivatives continuous for all real values of their arguments and are oscillatory in the following sense:

for each argument $u$ there exist such numbers $\beta_{1}>\alpha_{1}>u>\alpha_{-1}>\beta_{-1}$ that

$$
f\left(\alpha_{1}\right)<0, f\left(\beta_{1}\right)>0, f\left(\alpha_{-1}\right)<0, \quad f^{\prime}\left(\beta_{-1}\right)>0,
$$

where $f$ is either $h(x)$ or $p(t), u$ is either $x$ or $t$ and all roots of the restoring term $h(x)$ are isolated.

2. Our main tool for attacking the equation (1) will be the well-known Cauchy formula for the particular solution of nonhomogeneous linear differential equations with constant coefficients.

Lemma 1. If there exist such positive constants $H, P$ that for all $x \in \mathscr{R}^{1}$ and $t \geqq 0$ the inequalities

$$
\text { 1) }|h(x)| \leqq H, 2)\left|p^{\prime}(t)\right| \leqq P
$$

hold, then each solution $x(t)$ of the equation (1) satisfies the inequalities

$$
\begin{gathered}
\limsup _{t \rightarrow \infty}\left|x^{\prime}(t)\right| \leqq(H+P) / b:=D^{\prime}, \\
\limsup _{t \rightarrow \infty}\left|x^{\prime \prime}(t)\right| \leqq 2(H+P) / a:=D^{\prime \prime} .
\end{gathered}
$$

Proof. Substituting $y:=x^{\prime}$, we get from (1) the equation

$$
y^{\prime \prime}+a y^{\prime}+b y=p(t)-h(x(t))
$$


with solutions of the form

$$
\mid x^{\prime}(t)=/ y(t)=C_{1} \mathrm{e}^{\varrho_{1} t}+C_{2} \mathrm{e}^{\varrho_{2} t}+\int_{0}^{t} \frac{\mathrm{e}^{\varrho_{1}(t-\tau)}-\mathrm{e}^{\varrho_{2}(t-\tau)}}{\varrho_{1}-\varrho_{2}}[p(\tau)-h(x(\tau))] \mathrm{d} \tau,
$$

where $\varrho_{1,2}=\left(-a \pm \sqrt{ }\left(a^{2}-4 b\right)\right) / 2$ and $C_{1}, C_{2}$ are arbitrary constants.

Hence by virtue of 1$), 2$ ), for $t \geqq 0$ we have not only

$$
\left|\int_{0}^{t} \frac{\mathrm{e}^{\varrho_{1}(t-\tau)}-\mathrm{e}^{\varrho_{2}(t-\tau)}}{\varrho_{1}-\varrho_{2}}[p(\tau)-h(x(\tau))] \mathrm{d} \tau\right| \leqq \frac{H+P}{b}\left(1+\frac{\varrho_{2} \mathrm{e}^{\varrho_{1} t}-\varrho_{1} \mathrm{e}^{\varrho_{2} t}}{\varrho_{1}-\varrho_{2}}\right),
$$

but also

$$
\limsup _{t \rightarrow \infty}\left|x^{\prime}(t)\right| \leqq(H+P) / b .
$$

Furthermore, putting $z:=y^{\prime}$, we get from (3) the equation

$$
z^{\prime}+a z=p^{\prime}(t)-b x^{\prime}(t)-h(x(t))
$$

with solutions of the form

$$
\left|x^{\prime \prime}(t)=\right| z(t)=C \mathrm{e}^{-a t}+\int_{T_{x}}^{t} \mathrm{e}^{-a(t-\tau)}\left[p(\tau)-b x^{\prime}(\tau)-h(x(\tau))\right] \mathrm{d} \tau,
$$

where $C$ is an arbitrary constant and $T_{x}$ a great enough number.

Thus by virtue of 1 ), 2) and (4), for $t \geqq T_{x}$ we have not only

$$
\begin{gathered}
\left|\int_{T_{x}}^{t} \mathrm{e}^{-a(t-\tau)}\left[p(\tau)-b x^{\prime}(\tau)-h(x(\tau))\right] \mathrm{d} \tau\right| \leqq 2\left(H+P+\left|o\left(T_{x}\right)\right|\right) \int_{T_{x}}^{t} \mathrm{e}^{-a(t-\tau)} \mathrm{d} \tau \leqq \\
\leqq \\
a \\
\left.\qquad H+P+\left|o\left(T_{x}\right)\right|\right)\left(1-\mathrm{e}^{-a\left(t-T_{x}\right)}\right),
\end{gathered}
$$

but also

$$
\limsup _{t \rightarrow \infty}\left|x^{\prime \prime}(t)\right| \leqq 2(H+P) / a, \text { q.e.d. }
$$

Lemma 2. Under the assumptions of Lemma 1, if

$$
\left.\left|h^{\prime}(x)\right| \leqq H^{\prime} \text { for all } x \in \mathscr{R}^{1}, 3\right)\left|\int_{0}^{\infty} p(t) \mathrm{d} t\right|<\infty,
$$

where $H^{\prime}$ is a suitable constant, then every bounded solution $x(t)$ of the equation (1) either satisfies the relation

$$
\lim _{t \rightarrow \infty} x(t)=\bar{x}, \quad \lim _{t \rightarrow \infty} x^{\prime}(t)=\lim _{t \rightarrow \infty} x^{\prime \prime}(t)=0 \quad(h(\bar{x})=0)
$$

or there exists such a root $\bar{x}$ of $h(x)$ that $(x(t)-\bar{x})$ oscillates.

Proof. Substituting a fixed bounded solution $x(t)$ of (1) into (1) and integrating the result from $T_{x}$ to $t\left(T_{x}-\right.$ a great enough number, whose magnitude will be speci- 
fied later in (9)), we get the identity

$$
\begin{gathered}
\int_{T_{x}}^{t} h(x(\tau)) \mathrm{d} \tau=-\left\{b\left[x(t)-x\left(T_{x}\right)\right]+a\left[x^{\prime}(t)-x^{\prime}\left(T_{x}\right)\right]+x^{\prime \prime}(t)-x^{\prime \prime}\left(T_{x}\right)\right\}+ \\
+\int_{T_{x}}^{t} p(\tau) \mathrm{d} \tau(: \equiv I(t)) .
\end{gathered}
$$

Therefore, by virtue of the condition 3), the assertion of Lemma 1 and the boundedness of $x(t)$, there exists such a constant $M_{x}$ that for $t \geqq T_{x}$ the relation

$$
|I(t)| \leqq M_{x} \text { i.e. }\left|\int_{T_{x}}^{t} h(x(\tau)) \mathrm{d} \tau\right| \leqq M_{x}
$$

is satisfied.

Now let us assume that $x(t)$ does not converge to any root $\bar{x}$ of $h(x)$ : i.e.,

$$
\limsup _{t \rightarrow \infty}|x(t)-\bar{x}|>0
$$

and simultaneously, for $t \geqq T_{x}$,

$$
h(x(t)) \geqq 0 \text { or } h(x(t)) \leqq 0 .
$$

Then

$$
H(t): \equiv \int_{T_{x}}^{t} h(x(\tau)) \mathrm{d} \tau \quad\left(\text { for } t \geqq T_{x}\right)
$$

evidently is a composed monotone function with a finite or infinite limit for $t \rightarrow \infty$. Since (7) implies that the "divergent case" can be disregarded, it follows from (9) that not only

$$
\lim _{t \rightarrow \infty} \int_{T_{x}}^{t}|h(x(\tau))| \mathrm{d} \tau=\lim _{t \rightarrow \infty}\left|\int_{T_{x}}^{t} h(x(\tau)) \mathrm{d} \tau\right| \leqq M_{x}
$$

but also

$$
\liminf _{t \rightarrow \infty}|x(t)-\bar{x}|=0
$$

holds, because otherwise (i.e. if

$$
\left.\liminf _{t \rightarrow \infty}|x(t)-\bar{x}|>0\right)
$$

(9) together with the fact that the roots of $h(x)$ are isolated would yield

$$
\liminf _{t \rightarrow \infty}|h(x(t))|=\liminf _{t \rightarrow \infty}|h(x(t))-h(\bar{x})|>0,
$$

a contradiction to $\left(7^{\prime}\right)$.

Thus (8) and $\left(8^{\prime}\right)$ imply

$$
\limsup _{t \rightarrow \infty}|h(x(t))|=\limsup _{t \rightarrow \infty}|h(x(t))-h(\bar{x})|>0=\liminf _{t \rightarrow \infty}|h(x(t))|
$$

and consequently there exists such a sequence $\left\{t_{i}\right\} \geqq T_{x}$ and such a constant $\widetilde{H}>0$ 
that (in what follows, $\mathrm{d}(x, y)$ denotes the distance between $x$ and $y$ )

hold. Hence

$$
\text { a) } \left.\liminf _{i \rightarrow \infty / \Rightarrow \rightarrow t_{i} \rightarrow \infty /} \mathrm{d}\left(t_{i}, t_{i-1}\right)>0, \quad \beta\right)\left|h\left(x\left(t_{i}\right)\right)\right| \geqq \tilde{H}
$$

$$
\left.M_{x} \geqq \lim _{t \rightarrow \infty} \int_{t_{1}}^{t} \mid h_{(}^{\prime} x(\tau)\right)\left|\mathrm{d} \tau=\sum_{i=2}^{\infty} \int_{t_{i-1}}^{t_{i}}\right| h(x(\tau))\left|\mathrm{d} \tau \Rightarrow \limsup _{i \rightarrow \infty / \Rightarrow t_{i} \rightarrow \infty /} \int_{t_{i-1}}^{t_{i}}\right| h(x(t)) \mid \mathrm{d} t=0
$$

or $($ cf. $\alpha), \beta))$

$$
H^{\prime} \limsup _{t \rightarrow \infty}\left|x^{\prime}(t)\right| \geqq \limsup _{t \rightarrow \infty}\left|\frac{\mathrm{d} h(x(t))}{\mathrm{d} x(t)} x^{\prime}(t)\right|=\limsup _{t \rightarrow \infty}\left|\frac{\mathrm{d} h(x(t))}{\mathrm{d} t}\right|=\infty .
$$

But according to the assertion of Lemma 1, this is impossible and that is why $(x(t)-\bar{x})$ necessarily oscillates.

The remaining part of our lemma follows immediately from the assertion

$$
\begin{gathered}
\left.x(t) \in \mathbb{C}^{(n)}<0, \infty\right), \quad \limsup _{t \rightarrow \infty}\left|x^{(n)}(t)\right|<\infty, \\
\lim _{t \rightarrow \infty}|x(t)|<\infty \Rightarrow \lim _{t \rightarrow \infty} x^{(k)}(t)=0,
\end{gathered}
$$

(where $n \geqq 2$ is a natural number and $k=1, \ldots,(n-1)$ ),

whose proof can be found e.g. in [1, p. 161]. This completes the proof.

Lemma 3. Under the assumptions of Lemma 2 and if

$$
\left.\left.2^{\prime}\right) \quad\left|p^{\prime}(t)\right| \leqq P^{\prime} \quad \text { for all } t \geqq 0, \quad 2^{\prime \prime}\right) \limsup _{t \rightarrow \infty}|p(t)|>0
$$

hold, where $P^{\prime}$ is a suitable constant, then for every bounded solution $x(t)$ of the equation (1) there exists such a root $\bar{x}$ of $h(x)$ that $(x(t)-\bar{x})$ oscillates.

Proof. If Lemma 3 does not hold, then according to Lemma 2 (5) holds and the fourth derivative of $x(t)$ satisfies

$$
x^{\prime \prime \prime}(t)=p^{\prime}(t)-a x^{\prime \prime \prime}(t)-b x^{\prime \prime}(t)-h^{\prime}(x) x^{\prime}(t) .
$$

But it can be readily checked that, by the ultimate boundedness of $x^{\prime}(t), x^{\prime \prime}(t), x^{\prime \prime \prime}(t)$ (see (2)) and $\left.\left.1^{\prime}\right), 2^{\prime}\right)$, there exists such a constant $D_{4}$ that

$$
\limsup _{t \rightarrow \infty}\left|x^{\prime \prime \prime}(t)\right| \leqq D_{4},
$$

which according to $(10)$ gives the relations

$$
\lim _{t \rightarrow \infty} x(t)=\bar{x} \mid \Rightarrow \lim _{t \rightarrow \infty} h^{\prime}(x(t))=h(\bar{x})=0 /, \quad \lim _{t \rightarrow \infty} x^{(j)}(t)=0 \quad j=1,2,3
$$

or

$$
\limsup _{t \rightarrow \infty}|p(t)|=\limsup _{t \rightarrow \infty}\left|x^{\prime \prime \prime}(t)+a x^{\prime \prime}(t)+b x^{\prime}(t)+h(x(t))\right|=0,
$$

a contradiction to $\limsup _{t \rightarrow \infty}|p(t)|>0\left(\right.$ cf. $\left.2^{\prime \prime}\right)$ ), q.e.d. 
3. Now we can give the principal result of our paper.

Theorem. If there exist such positive constants $H, H^{\prime}, P, P^{\prime}, P_{0}, R$ that for $|x|>R$ and $t \geqq 0$ the following conditions are satisfied:

1) $|h(x)| \leqq H,\left|h^{\prime}(x)\right| \leqq H^{\prime}$,

2) $|p(t)| \leqq P,\left|p^{\prime}(t)\right| \leqq P^{\prime},\left|\int_{0}^{t} p(\tau) \mathrm{d} \tau\right| \leqq P_{0}, \limsup _{t \rightarrow \infty}|p(t)|>0$,

3) $\min \left[\mathrm{d}\left(\bar{x}_{k}, \bar{x}_{k+1}\right), \mathrm{d}\left(\bar{x}_{k}, \bar{x}_{k-1}\right)\right]>\frac{2(H+P)}{b}\left(\frac{2}{a}+\frac{a}{b}\right)+\frac{P_{0}}{b}$,

where $\bar{x}_{k}$ are roots of $h(x)$ with $h^{\prime}\left(\bar{x}_{k}\right)>0$ and $\bar{x}_{k-1}, \bar{x}_{k+1}$ denote the couple of adjacent roots of $\bar{x}_{k}(k=0, \pm 2, \pm 4, \ldots)$, then all solutions $x(t)$ of the equation (1) are bounded and for each of them there exists such a root $\bar{x}$ of $h(x)$ that $(x(t)-\bar{x})$ oscillates.

Proof. Let us assume, on the contrary, that $x(t)$ is an unbounded solution of (1); i.e., for example, lim sup $x(t)=\infty$.

Lemma 1 implies the existence of such a number $T_{0} \geqq 0$ great enough that for $t \geqq T_{0}$

$$
\left|x^{\prime}(t)\right| \leqq D^{\prime}+\varepsilon_{1}, \quad\left|x^{\prime \prime}(t)\right| \leqq D^{\prime \prime}+\varepsilon_{2},
$$

with $\varepsilon_{1}>0, \varepsilon_{2}>0$ small enough constants.

Let $T_{1} \geqq T_{0}$ be the last point with $x\left(T_{1}\right)=\dot{\bar{x}}_{k}\left(k\right.$-even) and $T_{2}>T_{1}$ be the first point with $x\left(T_{2}\right)=\bar{x}_{k+1}$. If we integrate (1) from $T_{1}$ to $t, T_{1} \leqq t \leqq T_{2}$, we come to

$$
\begin{gathered}
{\left[x^{\prime}(t)-x^{\prime \prime}\left(T_{1}\right)\right]+a\left[x^{\prime}(t)-x^{\prime}\left(T_{1}\right)\right]+b\left[x(t)-x\left(T_{1}\right)\right]+} \\
+\int_{T_{1}}^{t} h(x(\tau)) \mathrm{d} \tau=\int_{T_{1}}^{t} p(\tau) \mathrm{d} \tau .
\end{gathered}
$$

However, for $T_{1} \leqq t \leqq T_{2}$ we have $h(x(t)) \operatorname{sgn} x(t) \geqq 0$, whence we can obtain (multiplying (11) by $\operatorname{sgn} x$ )

$$
|x(t)| \leqq\left|x\left(T_{1}\right)\right|+\frac{2}{b}\left[D^{\prime \prime}+a D^{\prime}+\frac{1}{2} P_{0}\right]+\varepsilon,
$$

where $\varepsilon>0$ is an arbitrarily small constant, a contradiction to $x\left(T_{2}\right)=\bar{x}_{k+1}$ with respect to 3 ).

Since the remaining part of our theorem immediately follows from Lemma 3 , the proof is complete.

4. In the end, let us note that in [2] we have dealt also with the case

$$
\int_{0}^{\infty}|p(t)| \mathrm{d} t<\infty .
$$




\section{References}

[1] W. A. Coppel: Stability and Asymptotic Behavior of Differential Equations, D. C. Heath, Boston, 1975.

[2] J. Andres: Asymptotic properties of solutions of a certain third order differential equation with the oscillatory restoring term, to appear.

Author's address: 77146 Olomouc, Gottwaldova 15, Czechoslovakia (Joint Laboratory of Optics of Czech. Acad. Sci. and Palacký University). 